\section{C4 \\ Combined, the surface modification and quantum logic improvements result in a 500-fold sensitivity gain}

\title{
Probing proteins with diamond defects
}

Recent breakthroughs in NMR spectroscopy - traditionally limited to samples on a milligram scale - have brought the possibility of structural determination of individual molecules within sight. Specifically, defects in the lattice structure of diamond, called nitrogen-vacancy $(\mathrm{NV})$ centres, have been used as sensors or, more precisely, magnetometers to detect single nuclear spins at room temperature. Now, Mikhail Lukin, Hongkun Park and co-workers at Harvard University show that they can create NV centres with sufficient sensitivity to detect individual proton spins, which enables the analysis of individual protein molecules with NMR spectroscopy.

An NV centre in a diamond lattice is characterized by the replacement of two carbon atoms by a nitrogen atom and an adjacent vacancy. The spin states of these atomic defects can interact with the nuclear spins of atoms that are in close proximity; however, the lack of coherence of NV centres near the diamond surface has, to date, limited the effective use of these defects in spectroscopic techniques. Although longer coherence times require averaging to take place over slightly longer sampling periods, this is in fact desirable because the resulting increase in accumulated phase increases the intensity of the NMR signal.

"This study combines several new developments to improve the sensitivity of NV-based NMR spectroscopy," says Igor Lovchinsky, first author of the study. One such development is the modification of the diamond surface using wet oxidative chemistry and annealing at $465^{\circ} \mathrm{C}$. This process creates NV centres at shallow depths (between 3 and $6 \mathrm{~nm}$ ), resulting in improved spin coherence properties and thus enabling single-proton sensitivity after 1 second of averaging the output signal.

The protein of choice for this study is ubiquitin - a regulatory protein comprising 76 amino-acid residues. The proteins are covalently attached to the modified diamond surface using carbodiimide coupling chemistry. Atomic force microscopy images reveal that the ubiquitin molecules do not aggregate on the diamond surface and that the average spacing between individual proteins is greater than the typical depth of the NV centres. In addition to the surface modification method, the researchers further improved the sensitivity of the system using a two-qubit quantum system comprising an electronic spin and the ${ }^{15} \mathrm{~N}$ nuclear spin of the NV centre - the output of which is read using quantum logic. Combined, the surface modification and quantum logic improvements result in a 500 -fold sensitivity gain with respect to the researchers' findings when these two techniques are not implemented.

The researchers envisage that the enhanced sensitivity of the NMR spectroscopic analysis using NV centres will allow the dynamics of complex structures, such as individual biological molecules, to be probed. "The use of quadrupolar nuclear spins allows us to improve the spectral resolution of NV-based NMR spectroscopy and potentially allows individual spins to be spectrally resolved," says Lovchinsky. "Although these demonstrations allow the detection of individual molecules and some simple linewidth measurements, straightforward extensions of our method may provide a route to structure determination at the single-molecule level."

Alison Stoddart

ORIGINAL ARTICLE Lovchinsky, l. et al. Nuclear magnetic resonance detection and spectroscopy of single proteins using quantum logic. Science http://dx.doi.org/10.1126/science.aad8022 (2016)

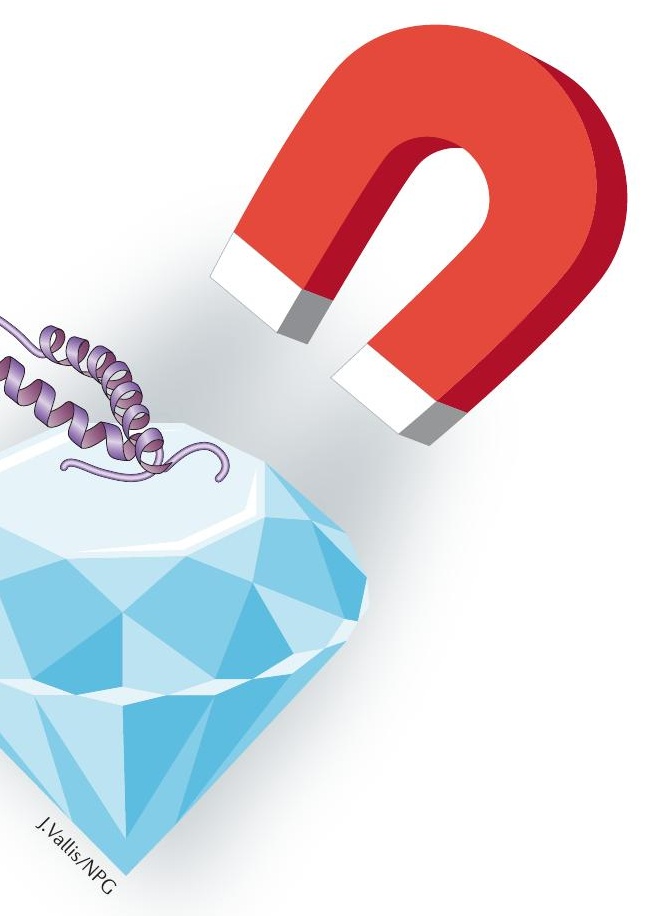

